\title{
Um passeio estatístico aplicado às medidas governamentais: incentivo a vacinação contra raiva em bovinos, na região central do estado Rondônia
}

A raiva é uma zoonose, uma doença infecciosa que afeta tanto seres humanos quanto animais, causando surtos clínicos e não tem cura. É considerada uma das zoonoses de maior importância em Saúde Pública, é transmitida pela mordida de um animal contaminado e/ou pela manipulação de animais doentes, ocorrendo à transmissão através da saliva. É uma ameaça ativa à saúde da população, especialmente em áreas rurais, onde há vários vetores para a sua transmissão. Sua relevância não é só nacional, mas também mundial, uma vez que tem gerado prejuízos incalculáveis à pecuária, acarretando grandes perdas econômicas. O presente estudo objetivou diagnosticar e analisar a variação da taxa de vacinação de bovinos contra raiva no município de Presidente Médici, estado de Rondônia nos anos de 2010 a 2015, a partir da incorporação de uma simples pergunta, como uma das formas de incentivo a vacinação. Desta forma, exibindo a efetividade desta medida de intervenção, adotado pelo governo do estado de Rondônia, com o intuito de estimular a vacinação contra raiva em bovinos. Para o levantamento das informações, buscou-se no banco de dados no Sistema de Informação da Agência IDARON-SISIDARON e de posse dessas informações, usando uma metodologia de cunho quantitativo/descritivo/documental, os dados foram analisados e demonstraram que, a partir de 2014, data da incorporação da medida, o nível de animais vacinados e declarados cresceram de forma abrupta, cerca de $144 \%$ em relação ao ano anterior, e no ano seguinte, houve outro crescimento, de $34,16 \%$, ao passo que o crescimento médio populacional/ano dos bovinos fo 3,16\%. Portanto, é importante mencionar a eficiência, eficácia e a efetividade desta medida, não só no município, mas também em todo o Estado de Rondônia, revelando como uma simples medida influenciou de maneira crucial a taxa de vacinação numa região, acarretando-se numa cobertura vacinal significativa comparada com a que existia anteriormente.

Palavras-chave: Efetividade; IDARON; Lyssavirus.

\section{A statistical walk applied to governmental measures: encourage vaccination against rabies in cattle, in the central region of the state of Rondônia}

Rabies is a zoonosis, an infectious disease that affects both humans and animals, causing clinical outbreaks and has no cure. It is considered one of the most important zoonoses in Public Health, it is transmitted by the bite of an infected animal and/or by the manipulation of sick animals, occurring to transmission through saliva. It is an active threat to the health of the population, especially in rural areas, where there are several vectors for its transmission. Its relevance is not only national, but also worldwide, since it has generated incalculable losses to livestock, causing great economic losses. The present study aimed to diagnose and analyze the variation in the rate of vaccination of cattle against rabies in the municipality of Presidente Médici, state of Rondônia in the years 2010 to 2015, from the incorporation of a simple question, as one of the forms of incentive to vaccination. Thus, showing the effectiveness of this intervention measure, adopted by the state government of Rondônia, in order to stimulate vaccination against rabies in cattle. To collect the information, we searched the database in the intervention measure, adopted by the state government of Rondônia, in order to stimulate vaccination against rabies in cattle. To collect the information, we searched the database in the
Information System of the IDARON-SISIDARON Agency and in possession of this information, using a quantitative/descriptive/documentary methodology, the data were analyzed and demonstrated that, from 2014, date of incorporation of the measure, the level of vaccinated and declared animals grew abruptly, about $144 \%$ in relation to the previous year, and in the following year, there was another growth, of $34.16 \%$, while the growth average population/year of cattle was $3.16 \%$. Therefore, it is important to mention the efficiency, efficacy and effectiveness of this measure, not only in the municipality, but also throughout the state of Rondônia, revealing how a simple measure has crucially influenced the vaccination rate in a region resulting in coverage significant vaccine compared to what previously existed.

Keywords: Effectiveness; IDARON; Lyssavirus.

Topic: Planejamento, Gestão e Políticas Públicas Ambientais

Reviewed anonymously in the process of blind peer.
Received: 02/01/2021 Approved: 29/01/2021
Rafael Luis da Silva (iD

São Lucas Educacional, Brasil

http://lattes.cnpq.br/3368127484992279

http://orcid.org/0000-0002-9485-479X

rafaeluismat@gmail.com

Flavia da Silva Nogueira

Universidade Federal de Viçosa, Brasil

http://lattes.cnpq.br/0680108586272405

rafaeluismat@gmail.com

Fabio Herrera Fernandes (iD)

Centro Universitário São Lucas, Brasil

http://lattes.cnpq.br/8154366932618987

http://orcid.org/0000-0003-3242-8303

fabio26012002@gmail.com
Referencing this:

SILVA, R. L.; NOGUEIRA, F. S.; FERNANDES, F. H.. Um passeio estatístico aplicado às medidas governamentais: incentivo a vacinação contra raiva em bovinos, na região central do estado Rondônia. Revista Ibero Americana de Ciências Ambientais, v.12, n.1, p.699706, 2021. DOI: http://doi.org/10.6008/CBPC21796858.2021.001.0056 


\section{INTRODUÇÃO}

A raiva é uma zoonose, uma doença infecciosa que afeta tanto seres humanos quanto animais, causando surtos clínicos e não tem cura (GREEN et al., 2006; BRASIL, 2015). É considerada uma das zoonoses de maior importância em Saúde Pública, não só por sua evolução drástica e letal, como também por seu elevado custo social e econômico (BRASIL, 2008; BRASIL, 2012).

Esta doença é transmitida pela mordida de um animal contaminado, e também através de manipulação de animais doentes, ocorrendo à transmissão através da saliva, Baptista et al. (2007), apesar de não haver tratamento, existe vacina para a prevenção (NOVAIS et al., 2008).

O vírus da raiva é do gênero Lyssavirus, da família Rhabdoviridae, que é o causador da doença (FEVRÉ et al., 2006), segundo o manual técnico de controle da raiva em herbívoros, BRASIL (2012) a família Rhabdoviridae está subdividida em dois subgrupos de vírus de plantas, um grupo de vírus de peixes e três grupos de vírus de mamíferos, este último correspondendo aos gêneros: Vesiculovirus, relacionado com doença vesicular em animais; Ephemerovirus, relacionado com a febre efêmera dos bovinos; Lyssavirus, relacionado com encefalomielite fatal em mamíferos. Este último pode infectar todos os animais de sangue quente, dentre estes, preferindo os mamíferos (BIGRAS-POULIN, 2006).

No Brasil, os morcegos hematófagos, em especial do gênero Desmodus rotundus, são os principais responsáveis pelo acometimento do vírus a herbívoros (BRASIL, 2017). São conhecidos 11 genótipos de vírus do gênero Lyssavirus, sendo o tipo 1 o único genótipo circulante no Brasil (CRMV-PR, 2010).

Conforme o Brasil (2012), no Brasil a principal espécie animal que transmite o vírus da raiva ao homem continua sendo o cão, embora os morcegos hematófagos estejam cada vez mais contribuindo para o aumentando de casos, podendo ser os principais responsáveis pela manutenção do vírus no ambiente silvestre (BRASIL, 2008). Várias são as identificações positivas do vírus da raiva que já foram confirmadas em animais silvestres no Brasil, como por exemplo, as raposas, jaritatacas, guaxinins, saguis, cachorro-do-mato e os morcegos hematófagos/Desmodus rotundus (BRASIL, 2008; CRMV-PR, 2010).

A raiva ainda é uma ameaça ativa à saúde da população, especialmente em áreas rurais, onde há vários pontos críticos para a transmissão, um dos vetores é a presença de morcegos hematófagos, e que uma vez identificado casos de mordeduras, poderá acarretar perdas inestimáveis tanto para a saúde pública como para a economia de uma região.

É importante frisar, que sua importância não é só nacional, mas também mundial, uma vez que tem gerado prejuízos incalculáveis à pecuária, gerando grandes perdas econômicas (GUITIÉRREZ, 2008; DIAS et al., 2011). É caracterizada por uma encefalomielite aguda e fatal, que pode acometer todos os mamíferos, apresentando variação de suscetibilidade a depender da espécie, (KEELING et al., 2005).

No Brasil a raiva dos herbívoros pode ser considerada endêmica e em graus diferenciados, de acordo com a região. Os principais fatores que contribuem para sua disseminação, ainda de forma insidiosa e preocupante, nos herbívoros domésticos são: aumento da oferta de alimento, representado pelo significativo crescimento dos rebanhos; ocupação desordenada, caracterizada por macro modificações ambientais, como 
Um passeio estatístico aplicado às medidas governamentais: incentivo a vacinação contra raiva em bovinos, na região central do estado

desmatamento, construção de rodovias e de hidrelétricas, que alteraram o ambiente em que os morcegos viviam, obrigando-os a procurar novas áreas e outras fontes de alimentação; oferta de abrigos artificiais, representados pelas construções, como túneis, cisternas, casas abandonadas, bueiros, fornos de carvão desativados e outros; atuação insatisfatória, em alguns estados brasileiros, na execução do Programa Estadual de Controle da Raiva dos Herbívoros.

Com isso vem à tona a seguinte pergunta, 'é possível obter por meio de medidas governamentais a estimulação à vacinação de bovinos, contra raiva, na região central do estado de Rondônia, pelos produtores rurais?'. As contribuições que esta pesquisa pode trazer é apropriação de respostas aos problemas citados anteriormente, ou em contrapartida, ampliar as formulações teóricas a esse respeito. Prevenir, controlar e/ou erradicar enfermidades é necessário e que deve envolver toda a cadeia da defesa sanitária, León et al. (2006). O presente estudo objetivou diagnosticar e analisar a variação da taxa de vacinação de bovinos contra raiva no município de Presidente Médici, estado de Rondônia nos anos de 2010 a 2015, a partir da incorporação de uma simples pergunta, como uma das formas de incentivo a vacinação.

\section{METODOLOGIA}

O presente estudo foi desenvolvido com dados cedidos pela Agência de Defesa Sanitária Agrosilvopastoril do Estado de Rondônia - IDARON, referente ao município de Presidente Médici, estado de Rondônia, a partir da quantificação da população bovina e do número de animais vacinados contra raiva, com o objetivo de avaliar a efetividade e o grau de crescimento da taxa de animais vacinados. Trata-se de um estudo observacional, retrospectivo, com enfoque na quantificação dos registros. $O$ estudo teve como base os anos de 2010 a 2015.

Presidente Médici é um município brasileiro do interior do estado de Rondônia. Localiza-se a uma latitude -1110'33" sul e a uma longitude -61ำ5'03" oeste, estando a uma altitude de 185 metros em relação ao nível do mar e possui uma área territorial de 1.758 km² (IBGE, 2017), figura 1.

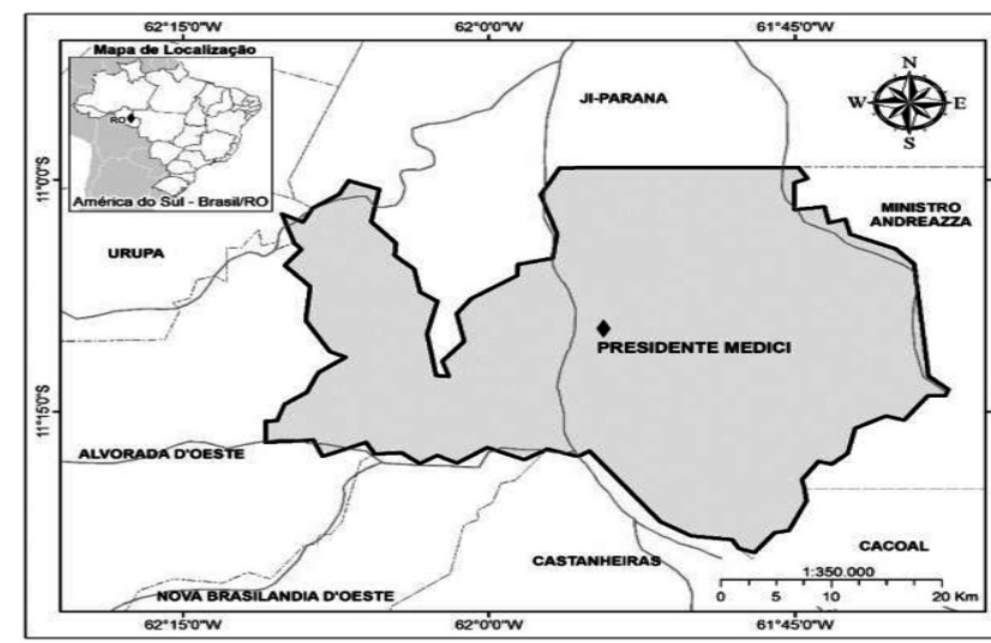

Figura 1: Mapa de localização, com destaque para a cidade de presidente Médici, Rondônia. Fonte: Santos et al. (2014).

Quanto os aspectos metodológicos, o presente trabalho caracterizou-se como uma pesquisa 
Um passeio estatístico aplicado às medidas governamentais: incentivo a vacinação contra raiva em bovinos, na região central do estado

documental, com abordagem de pesquisa quantitativa e característica descritiva, com dados discretos, sendo uma forma de estar buscando um número maior de informações e uma melhor aplicabilidade. Além de se ter um caráter quantitativo e descritivo, possui uma vertente de pesquisa documental, que pode ser caracterizada como uma pesquisa qualitativa, constituída do exame de materiais que ainda não receberam um tratamento analítico ou que podem ainda ser reexaminados com vistas a uma nova compreensão ou uma complementação do que se estava estudando anteriormente (SEVERINO, 2002; COELHO et al., 2008).

A pesquisa documental oferece uma base útil para outros tipos de estudos qualitativos, possibilitando que a criatividade do pesquisador dirija sua investigação por enfoques diferenciados (SEVERINO, 2002).

\section{Análise estatística}

Após coletados as informações através do banco de dados do SISIDARON, Sistema de Informações da Agência IDARON, os mesmos foram tabulados em planilhas eletrônicas e posteriormente utilizados na confeç̧ão de gráficos, visando uma melhor compreensão da distribuição destes, ao longo dos anos. Ainda, os mesmos foram submetidos à estatística descritiva, para identificação das frequências, absoluta e relativa, e, por conseguinte na utilização dos cálculos de média.

No que concerne aos dados relacionados à série temporal das vacinações, contra raiva, foi desenvolvido um gráfico de controle (GC), que segundo Vieira (2014), serve para identificar a variabilidade de certo conjunto de dados, monitorando se o processo está em controle, de tal modo que causas especiais de variação sejam identificadas. O GC é baseado na ideia de que a variabilidade se mantém numa faixa estável, e as observações futuras podem ser previstas através de dados anteriores, existentes dentro de limites de probabilidades estimadas (PYLRO, 2008). A representação do GC é realizada por três linhas denominadas, linha central (LC, que representa a média dos valores encontrados da característica investigada), e as linhas do limite superior de controle (LSC) e do limite inferior de controle (LIC), estas dispostas simetricamente em relação à LC (PYLRO, 2008).

Para a detecção dos LSC e LIC, foram utilizadas as inequações 1 e 2, para a identificação de anormalidades (outliers) nos valores das vacinações. As inequações foram baseadas no método de Barnett et al. (1994) que provém da Análise Exploratória de Dados (AED) para um conjunto de dados univariados (MONTGOMERY et al., 2003; COSTA et al., 2004; MONTGOMERY, 2004).

$$
\begin{array}{lr}
\text { O.I. }<Q 1-1,5 *(Q 3-Q 1) & \text { Inequação (1) } \\
\text { O.S. }>Q 3+1,5^{*}(Q 3-Q 1) & \text { Inequação (2) }
\end{array}
$$

Em que,

O.I. = outlier inferior; $O . S .=$ outlier superior; $Q 1=$ primeiro quartil; $Q 2=$ segundo quartil = mediana; $Q 3=$ terceiro quartil, (Q3-Q1) = amplitude interquartil.

Um quartil constitui $1 / 4$ dos dados amostrados, onde o primeiro quartil (quartil inferior, Q1) corresponde a $25 \%$ do total do conjunto de dados. O segundo quartil (Q2) corresponde ao valor da mediana onde $50 \%$ da amostra está ordenada. O terceiro quartil ou quartil superior (Q3) é o valor que delimita os $25 \%$ dos valores mais elevados, equivalentes a $75 \%$ do valor amostral, Fonseca et al. (2006). 


\section{RESULTADOS E DISCUSSÃO}

Durante o período, foi percebido, uma tendência de aumento, para todos os anos de estudo, quando relativizado de um ano para o outro, a saber: 2011 em relação a 2010 - população bovina cresceu 0,21\%; 2012 em relação a 2011- crescimento 1,46\%; 2013 em relação a 2012 - um novo aumento, de 0,87\%; 2014 em relação a 2013 - um incremento de 4,12\%; e finalmente em 2015 em relação a 2014 - população bovina cresceu 4,03\%. Representando um crescimento médio, para todo o período, de aproximadamente de 3,14\%, conforme exibido na figura 2.

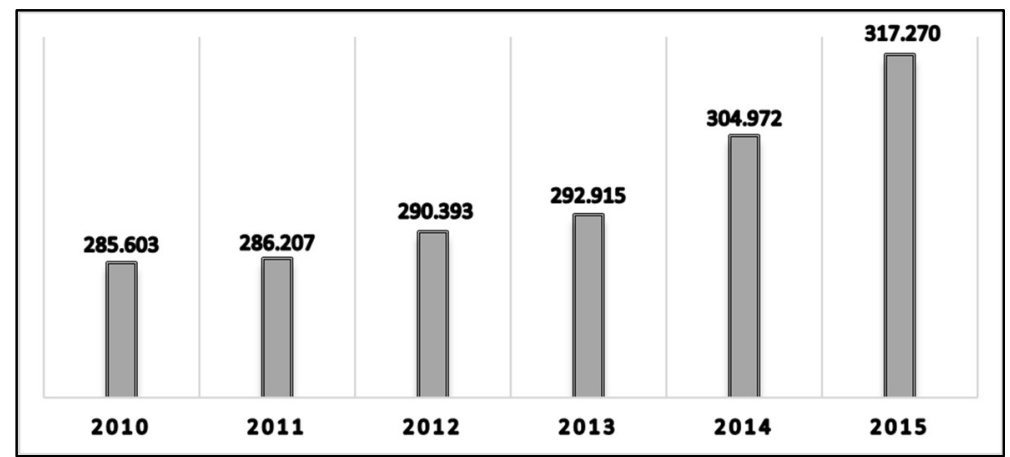

Figura 2: Série temporal do número de bovinos registrado no município de Presidente Médici, nos anos de 2010 a 2015.

Considerando o total de animais vacinados, durante o período amostral, observa-se uma média anual de 27.837 animais imunizados (Figura 3). A variação temporal do conjunto dos dados concomitantemente com os limites inferior e superior dos valores associados às vacinações exibiram para os anos de 2014 e 2015 os maiores valores de vacinações, que oscilaram em 41.198 a 55.253 animais vacinados, respectivamente. Tais valores estiveram acima da média anual (27.837), no entanto não foi considerado outliers da série temporal, todavia ficou nas proximidades (ano de 2015) de se apresentar como anormalidade da série (Figura 2).

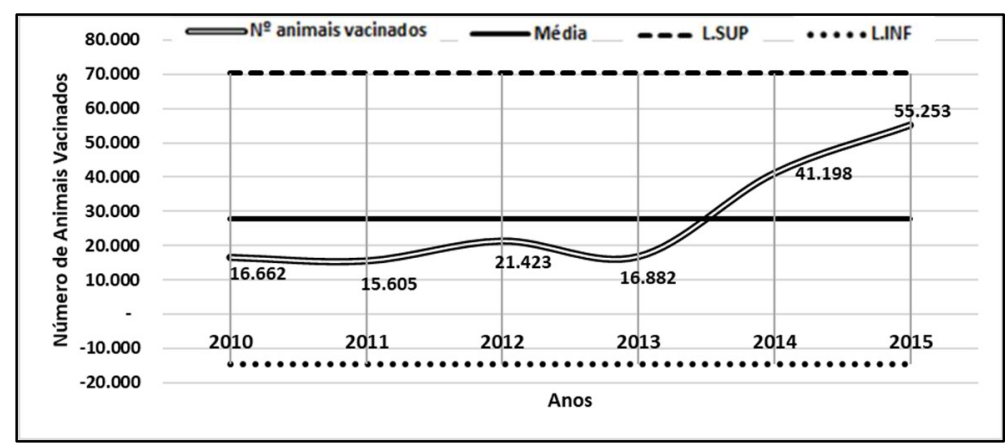

Figura 3: Análise da distribuição temporal das vacinações, contra raiva ocorridos no município de Presidente MédiciRO, no período de 2010 a 2015. Indicando os valores de média, limite inferior e superior da série de dados.

Quando confrontado as séries (quantitativo populacional de bovinos $\mathrm{x}$ animais vacinados), é detectado dois momentos cruciais. No primeiro momento (2010 a 2013) é identificado que o único ano que o número de vacinação não apresentou crescimento, em relação ao ano anterior, foi ano de 2011 em relação a 2010. No entanto o número populacional de bovino apresentou valores, sempre crescente, mesmo sendo de baixa intensidade (figura 4). 
Um passeio estatístico aplicado às medidas governamentais: incentivo a vacinação contra raiva em bovinos, na região central do estado

O segundo momento (2014 a 2015), é caracterizado pela data da incorporação da medida de incentivo à vacinação contra raiva, e decorreram as maiores taxas de vacinação, como também dos maiores índices de aumento populacional, sendo que: 2014 em relação a 2013 - população bovina cresceu 4,12\%, ao passo que a quantidade de animais vacinados aumentou em 144,03\%; 2015 em relação a 2014 - população bovina cresceu $4,03 \%$, ao passo que a quantidade de animais vacinados expandiu em $34,16 \%$ (figura 4 ).

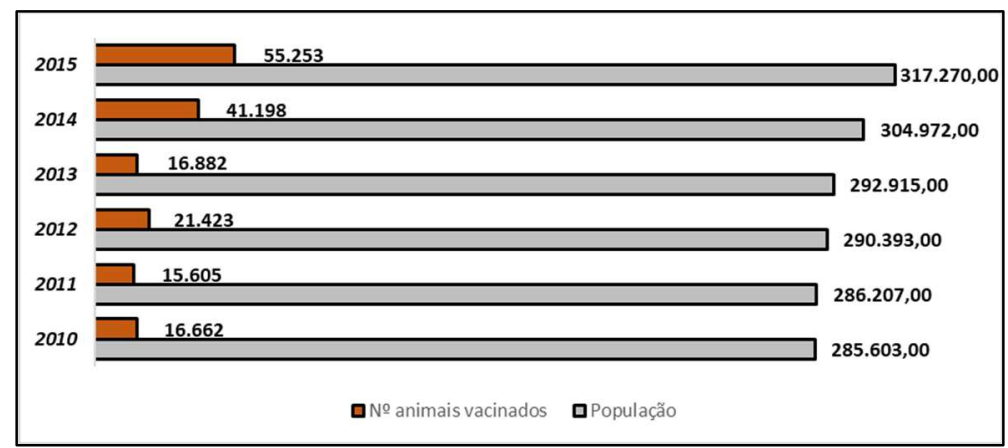

Figura 4: Série temporal confronto (número de bovinos x quantidade de bovinos vacinados contra raiva), em de Presidente Médici nos anos de 2010 a 2015.

É de importância mencionar, que o incentivo à vacinação contra essa enfermidade, foi inserido nas campanhas contra febre aftosa, como uma simples pergunta: 'vacinou contra raiva?'; o porquê de se incorporar essa pergunta em campanhas contra febre aftosa, decorre do fato, que é nessa época que a totalidade dos produtores vão declarar e atualizar o seu rebanho junto a IDARON e por causa dessa ação, foi obtido um significativo aumento no índice vacinal no município.

O mero questionamento sobre a vacinação do rebanho bovino durante a declaração de vacinação contra Febre Aftosa, deu margem a conversação com os produtores rurais sobre a necessidade de proteger seu rebanho através da vacinação, mesmo essa não sendo obrigatória na região.

Ao serem inqueridos pelos funcionários, os produtores perguntavam se era obrigatório e qual a necessidade deles em vacinar, momento em que foram instruídos da importância da vacinação, dos prejuízos e agravos da doença, do risco para a saúde humana, sobre a notificação de animais doentes, ataques de morcegos entre outras informações que surgiam durante essa conversa. A vacinação é compulsória quando da ocorrência de focos da doença e deve ser adotada preferencialmente em bovídeos e equídeos (BRASIL, 2012).

Porquanto, a Instrução Normativa № 5, de $1^{\circ}$ de março de 2002, preconiza que a vacinação dos herbívoros seja realizada com vacina contendo vírus inativado. A vacinação compulsória, não relacionada a foco, deverá ter caráter temporário, mantendo-se até que os programas estaduais atinjam níveis satisfatórios do controle da raiva (COELHO et al., 2008).

Essa medida teve início na 36a campanha contra febre aftosa, no ano de 2014, e como verificada na figura 3, houve um crescimento significativo de animais vacinados, cerca de 41.198 bovinos vacinados e cadastros junto a IDARON, em termos de percentagem representou um crescimento em torno de $144 \%$ em relação ao ano de 2013. Consequentemente no ano de 2015 foi representado outro aumento da taxa de vacinação, registrando assim, a quantidade de 55.253 animais vacinados. 
Um passeio estatístico aplicado às medidas governamentais: incentivo a vacinação contra raiva em bovinos, na região central do estado

É importante citar que os níveis de crescimento da população bovina nesses anos supracitados ocorreram de maneira um pouco variável, quanto se comparada com a quantidade de animais vacinados contra raiva, contudo o crescimento populacional dos animais já obedece a essa natureza de crescimento, porém o aumento ou a diminuição da taxa de vacinação contra raiva decorre de focos de raiva em municípios circunvizinhos, como é observado um aumento de vacinação nos anos de 2010 e 2012, ou de medidas intervencionistas adotadas pelo estado, através da agência IDARON.

Conforme discutido, vem se revelando, como uma pequena ação pode influenciar de maneira abrupta a taxa de vacinação em certa região. No ano de 2015 , foram imunizados $17,41 \%$ da totalidade dos bovinos do município, essa porcentagem de vacinação é consideravelmente relevante para a cidade de Presidente Médici, fazendo com que ocorra uma inibição da circulação do vírus da raiva.

\section{CONCLUSÕES}

Constatou-se que antes da incorporação da medida, a taxa de vacinação oscilava quase constante e não alcançava números significativos de vacinação, se comparada com o total de bovinos para a época. A partir de 2014 a taxa de vacinação cresceu de forma agressiva, aproximadamente 144\% em relação ao ano anterior. Já no ano de 2015, houve novamente, outro crescimento em relação a 2014, sendo de aproximadamente de $34 \%$. É importante mencionar que essa medida, foi realizada em todo o Estado de Rondônia, porém o estudo foi por hora realizada apenas no município de Presidente Médici.

Indubitavelmente, constata-se a efetividade dessa ação, que influenciou a taxa de vacinação na região, contribuindo com grande impacto para a imunização de bovinos, é importante mencionar, que tal ação não houve gasto financeiro para sua incorporação e a continuação desta. Tão importante o incentivo a vacinação é o fato dos órgãos responsáveis trabalharem com programas rotineiros, oferecendo desta forma, cursos que viabilizem ao produtor rural o incentivo à notificação de doenças, junto aos órgãos de defesa sanitária, trabalhando deste modo, não com a enfermidade identificada, através dos seus sinais clínicos, e sim com métodos preventivos de combate.

\section{REFERÊNCIAS}

BARNETT, V.; LEWIS, T.. Outliers in statistical data. 3 ed. New York: John Wiley \& Sons, 1994.

BAPTISTA, F. M.; NUNES, T.. Spatial analyses of cattle movement patterns in Portugal. Veterinaria Italiana, v.43, n.3, p.611-619, 2007.

BIGRAS-POULIN, M.; THOMPSON, R. A.; CHRIEL, M.. Network analysis of Danish cattle industry trade patterns as an evaluation of risk potential for disease spread. Preventive Veterinary Medicine, v.76, n.1, p.11-39, 2006.

BRASIL. Ministério da Agricultura, Pecuária e Abastecimento. Controle da Raiva em Herbívoros: Manual técnico. Brasília: MAPA/ACS, 2012.

BRASIL. Ministério da Agricultura, Pecuária e Abastecimento.
Revisão sobre raiva. Brasília: Comitê Científico Consultivo sobre Raiva, 2017.

BRASIL. Ministério da Saúde. Raiva: manual de diagnóstico laboratorial. Brasília: SAS, 2008.

BRASIL. Ministério da Saúde. Boletim Epidemiológico. Situação Epidemiológica de ocorrência de microcefalias no Brasil, 2015. Brasília: MDS, 2015.

COELHO, F. C.; CRUZ, O. G.; CODEÇO, C. T.. Epigrass: a tool to study disease spread in complex networks. Source Code Biol. Med., v.3, p.1-10, 2008.

COSTA, A. F. B.; EPPRECHT, E. K.; CARPINETTI, L. C. R. Controle estatístico da qualidade. São Paulo: Atlas, 2004. 
CRMV-PR. Conselho Regional de Medicina Veterinária do Estado do Paraná. Programa de Zoonoses da Região Sul. Manual de Zoonoses. Curitiba, 2010.

DIAS, R. A.; NOGUEIRA, F. V. S.; GOULART, C. S.; TELLES, I. C. O.; MARQUES, G. H. F.; FERREIRA, F.. Modelo de risco para circulação do vírus da raiva em herbívoros no Estado de São Paulo, Brasil. Rev. Panam. Salud. Public., v.30, n.4, p.370376, 2011.

GREEN, D. M.; KISS, I. Z.; KAO, R. R.. Modelling the initial spread of foot-and mouth disease through animal movements. Proceedings of the Royal Society B: Biological Sciences, v.273, n.1602, p.2729-35, 2006. DOI: http://doi.org/10.1098/rspb.2006.3648

FEVRÉ, E. M.; BRONSVOORT, B. M. C.; HAMILTON, K. A. Animal movements and the spread of infectious diseases. TRENDS in Microbiology, v.14, n.3, p.125131, 2006.

FONSECA, J. S.; MARTINS, G. A.. Curso de estatística. São Paulo: Atlas, 1996.

FONSECA, J. S.; MARTINS, G. A.. Curso de estatística. 6 ed. São Paulo: Atlas, 2006.

GUITIÉRREZ, H. O. D.. Formas de produção pecuária e distribuição da febre aftosa no departamento de Santa Cruz, Bolívia, 2000-2007. Tese (Doutorado em Medicina Veterinária) - Universidade Federal de Minas Gerais, Belo Horizonte, 2008.

IBGE. Instituto Brasileiro de Geografia e Estatística. Perfil das cidades brasileiras. Brasília: IBGE, 2017.
KEELING, M. J.; EAMES, K. T. D.. Networks and epidemic models. Journal of the Royal Society Interface, v.2, n.4, p.295-307, 2005.

LEÓN, E. A.; STEVENSON, M. A.; DUFFY, S. J.. A description of cattle movements in two departments of Buenos Aires province, Argentina. Prev. Vet. Med., v.76, p.109-120, 2006.

MONTGOMERY, D. C.; RUNGER, G. C.. Estatística aplicada e probabilidade para engenheiros. 2. d. Rio de Janeiro: LCT, 2003.

MONTGOMERY, D. C.. Introdução ao controle estatístico da qualidade. 4 ed. Rio de Janeiro: LCT, 2004.

NOVAIS, B. A. F.; ZAPPA, V.. Raiva em Bovinos: Revisão de Literatura. Revista Cient. de Medicina Veterinária, v.7, n.10, 2008.

PYLRO, A. S.. Modelo linear dinâmico de Harrison \& Stevens aplicado ao controle de processos auto-correlacionados. Tese (Doutorado em Engenharia de Produção) - Pontifícia Universidade Católica do Rio de Janeiro, Rio de Janeiro, 2008.

SANTOS, B. L.; ANDRADE, J. E.; SOUSA, R. G. C.. Densidade de estocagem utilizada no desenvolvimento do tambaqui em fase de pré-engorda. Scientia Amazônia, v.3, n.3, p.41-50, 2014.

SEVERINO, A. J.. Metodologia do trabalho científico. São Paulo: Cortez, 2002.

VIEIRA, S.. Estatística para a qualidade. 3 ed. Rio de Janeiro: Campus, 2014.

A CBPC - Companhia Brasileira de Produção Científica (CNPJ: 11.221.422/0001-03) detém os direitos materiais desta publicação. Os direitos referem-se à publicação do trabalho em qualquer parte do mundo, incluindo os direitos às renovaç̃̃es, expansões e disseminações da contribuição, bem como outros direitos subsidiários. Todos os trabalhos publicados eletronicamente poderão posteriormente ser publicados em coletâneas impressas sob coordenação da Sustenere Publishing, da Companhia Brasileira de Produção Científica e seus parceiros autorizados. Os (as) autores (as) posteriormente ser publicados em coletâneas impressas sob coordenação da Sustenere Publishing, da Companhia Brasileira de Produção Científica e seus parce
preservam os direitos autorais, mas não têm permissão para a publicação da contribuição em outro meio, impresso ou digital, em português ou em tradução. 